\title{
Productions alimentaires et productions non alimentaires : compétition ou complémentarité ?
}

Jean-Paul JAMET

Centre national pour la promotion des produits agricoles et alimentaires,

9 rue de la Baume, 75008 Paris

<gisele.michelet@cnpa.asso.fr>

\begin{abstract}
The topic is intentionally controversial. Prices on a number of food types used for biofuel have doubled in last couple of years. In the same time oil price is soaring. Many factors are contributing to the rise of food prices: the biggest is escalating demand. In recent years the economies of the world developing countries have been growing about 7 percent a year; other factors are droughts, new deregulation in agricultural policies (less intervention lowering stock levels), US dollar weakness benefiting commodity prices as safe haven asset and increase production costs like energy inputs. The explanation of the increases of oils prices are very close: the growth of new developing countries, political instability in fuel exporting countries, the loss of faith in US currency, the low investments in prospecting and refining of fuel. The use of renewable biofuels in lieu of fuel to reduce greenhouse gas emissions and increase energy security. Brazil produces 5 billions gal (19 millions $h \mathrm{~L}$ ) of sugarcane ethanol, enough to supply $45 \%$ of its transportation fuel demands. This production needs 6 millions hectares on a total of 400 millions ha for farming. In EU the proportion area devoted to biofuels is $1 \%$. In US, at the same time substantial price rises for maize, the main raw material for the US ethanol. The expected reduction in US maize exports would weigh heavily on the already tight supply on international grain market. In any event, even if maize yields were to achieve the require increase, the United States might have to limit exports in order to achieve its ambitious biofuels targets. The competition between food uses and biofuels is depending of the speed of development of second generation cellulosic and biomass based biofuels and of the purpose of US authorities to improve the level of grains stocks as corn, wheat and soya.

Key words: food price, food use, energy prices, biofuel
\end{abstract}

autant que l'Europe avec ses 500 millions d'habitants. Et donc, même si une bonne partie du monde rural chinois et du monde rural indien est à un niveau de pauvreté relativement important - beaucoup ne gagnent que 1 à 2 dollars par semaine - l'apparition d'une classe moyenne crée un choc en termes de demande sur le marché des produits agricoles.

Dans le même ordre d'idée, le renchérissement relativement récent du prix de l'énergie pétrolière et gazière fait qu'un certain nombre de pays qui ont des ressources pétrolières se portent beaucoup plus volontiers sur le marché des produits agricoles.

La deuxième raison de cette augmentation tient aux problèmes climatiques apparus au cours de ces dernières années dans des zones productrices de blé en particulier. L'Australie devient structurellement semi-désertique : une équipe du CNRS l'a démontré il n'y a pas longtemps, la déforestation de l'Amazonie conduit à charger moins en eau les alizés sur le Pacifique, si bien qu'El Nino contribue désormais à assécher toute une partie de l'Australie où la production de céréales est passée de 50 millions de tonnes à 15 millions de tonnes en 2006-2007. Par ailleurs, la sécheresse a touché également d'autres pays producteurs de céréales comme l'Ukraine, le Maroc, l'Espagne. Ces récoltes moins abondantes que prévu des zones traditionnellement productrices ajoutées à un accroissement de la demande, n'ont pu qu'agir sur le cours des produits agricoles et cela malgré l'augmentation des productions, en Amérique Latine, avec l'Argentine et le Brésil.

La troisième raison qu'il convient de mettre en avant, nettement plus structurelle que la précédente et très conséquente, renvoie à l'abandon des politiques agricoles de régulation des marchés, entériné par I'Uruguay Round. En particulier il n'y a plus de politique de régulation des stocks et cela a un effet très important au niveau des opérateurs - on ne dispose plus que de deux mois de stock en matière de production céréalière du fait d'un petit désajustement entre l'augmentation insuffisante de l'offre par rapport à la demande. Ces 5-6 dernières années a été constaté un écart de seulement de $1 \%$ en moyenne annuelle entre la consommation et la demande, ce qui naturellement s'est traduit par une baisse significative des stocks.

Quatrième explication de cette hausse des coûts des productions agricoles : la spéculation financière. Si on regarde le marché des commodités sur la longue durée, on constate une baisse générale tendancielle des prix qui s'étend jusque vers les années 2003-2004. Et en quatre ans, le prix de toutes les matières premières a doublé, qu'elles soient métallurgi- 
ques, énergétiques ou agricoles. II faut voir dans ce retournement de tendance un phénomène bien connu de tous les économistes, à savoir que la mauvaise monnaie chasse la bonne : vu l'incertitude sur le dollar, les opérateurs préfèrent placer leur argent sur les marchés des matières premières dont la tendance est haussière. La venue des fonds d'investissement et les fameux hedge funds sur les marchés des commodités n'ont fait qu'accentuer cette hausse. On a là une amplification liée à l'instabilité des monnaies et aux mouvements de masses financières cherchant les meilleurs placements possibles, sans doute dans une vision à très court terme.

Le dernier facteur enfin poussant à la hausse est celui des coûts de production. En réalité je ne crois pas à une augmentation démesurée du prix de l'énergie. On confond ici deux choses très différentes : la volatilité des prix et la tendance des marchés. Si l'on regarde sur une longue période, le prix de l'énergie au cours du $X X^{\mathrm{e}}$ siècle a été à peu près stable en monnaie constante. L'énergie est le résultat d'une production comme une autre et l'humanité y consacre à peu près $10 \%$ de ses capacités de production. Sans revenir à Jean-Baptiste Say, quelque part il y a un équilibre: l'énergie ne peut pas augmenter sans influencer le reste de l'économie. Donc naturellement, lorsqu'une hausse de l'énergie apparaît, elle se répercute en particulier sur les secteurs qui incorporent beaucoup d'énergie - et l'agrochimie avec la production d'engrais et le secteur agricole avec les travaux de labour et de récolte sont de gros consommateurs d'énergie. L'augmentation des coûts de production en agriculture est ainsi la conséquence pour partie de l'augmentation du coût de l'énergie et ne peut que se répercuter sur les cours des produits agricoles en pesant sur la capacité des opérateurs agricoles à augmenter leur offre.

\section{Les coûts de l'énergie}

II semble bien que les facteurs pesant sur l'évolution des prix des produits pétroliers présentent de nombreuses similitudes avec ceux déterminant l'évolution des prix agricoles.

Il y a tout d'abord à l'origine de cette hausse un facteur économique lié à l'évolution de la demande. Le décollage de la faculté productrice de I'Asie avec la Chine et I'Inde, mais aussi du Brésil, exerce désormais une pression durable sur la demande de produits pétroliers. Ceci dans un contexte marqué par une politique de maîtrise de l'offre de la part des pays producteurs et par une relative faiblesse des investissements en exploration et en raffinage notamment aux États-Unis: depuis une vingtaine d'années, ces derniers n'investissent plus dans ce secteur alors que la demande d'essence continue d'augmenter ${ }^{1}$.

Intervient également quant à cette hausse un facteur géopolitique : les réserves de pétrole et de gaz sont concentrées dans des pays politiquement peu stables (Iran, Irak, Nigeria, Angola, Venezuela...). L'instabilité du MoyenOrient est une menace permanente sur la sécurité des approvisionnements et des cours.

Une troisième raison est de nature financière : la spéculation et l'intervention sur ce marché des fonds d'investissement amplifient de manière significative les variations de prix. On estime qu'elle explique au moins $25 \%$ de la hausse actuelle des cours du pétrole.

Ceci dans un contexte où les réserves actuelles connues, l'amélioration des techniques d'extraction, les réserves de gaz et de schistes bitumineux assurent un siècle de consommation mondiale à technologie constante (rappelons les réserves de charbon: deux siècles de consommation, et d'uranium : un siècle). II n'y a donc pas de raison de fond à la hausse des produits énergétiques si ce n'est qu'ils relèvent de politiques de long terme alors qu'à I'heure actuelle les opérateurs financiers se déterminent dans le monde entier sur des politiques de court terme. Les opérateurs du secteur énergétique $n$ 'investissent pas facilement, si bien que la tension actuelle résulte plus d'une politique d'ajustement qu'elle ne dénote un manque potentiel de ressources.

La contrainte qui vient heurter ce secteur est le fameux problème du réchauffement climatique, contrainte qui milite en faveur de l'usage des biocarburants, source de carbone renouvelable.

\section{L'intérêt du recours aux biocarburants}

Dans un contexte marqué par la lutte contre le réchauffement climatique et la recherche de réduction des émissions de gaz à effet de serre, les biocarburants présentent l'intérêt de recourir à des sources de carbone renouvelables et donc d'éviter l'accroissement de l'effet de serre par l'emploi de carbone fossile.

Par ailleurs, ils permettent de diversifier les approvisionnements et indirectement de diminuer la pression sur la ressource pétrolière, raison également importante pour laquelle un certain nombre de pays se sont lancés dans leur production - les États-Unis, le Brésil, la Chine, I'Inde, I'Union européenne.

Une raison technique souvent oubliée préside également à leur adoption. Quelles que soient les discussions sur les rendements énergéti-

\footnotetext{
${ }^{1}$ Qui se souvient qu'en 1999 , le cours du baril de pétrole est tombé à 11 \$ ?
}

ques, les biocarburants d'aujourd'hui - biodiesel ou éthanol - sont mélangeables aux essences et gasoils actuels sans modifications importantes des flottes de transport. Des solutions autres qui exigeraient des motorisations différentes poseraient de tout autres problèmes d'investissement.

Néanmoins les biocarburants ne sont pas la réponse à l'épuisement des ressources fossiles de pétrole et de gaz. Les surfaces nécessaires à une substitution significative de carburants fossiles sont hors de portée : s'il fallait se fonder sur les productions végétales pour couvrir la demande mondiale en carburants d'ici 2050, la totalité des surfaces agricoles utiles n'y suffirait pas. La première politique de maîtrise de production de carburants - I'Agence internationale de l'Énergie ne cesse de le rappeler - réside dans les économies d'énergie.

Avec une consommation énergétique mondiale du secteur des transports de 1,6 milliard de tonnes d'équivalent pétrole (TEP), les 44 millions de TEP apportés par les biocarburants ne représentent au mieux que $3 \%$ du problème énergétique (figure 1)!

\section{Hausse des prix des produits agricoles et biocarburants}

La hausse des prix des produits agricoles estelle liée au prélèvement en matières premières appelé par la production de biocarburants?

Rappelons quelques données.

\section{Surfaces et volumes de production}

La production mondiale d'éthanol en 2005 (figure 2) s'est élevée à $37 \mathrm{Mt}$ essentiellement fabriquée à partir de canne à sucre au Brésil et de maïs aux États-Unis. La production brésilienne de cannes à sucre occupe $6 \mathrm{M}$ d'hectares alors que la surface des exploitations agricoles au Brésil est de $400 \mathrm{M}$ d'hectares: autrement dit, aujourd'hui au Brésil il n'y a pas de compétition sur le foncier entre productions énergétiques et production alimentaire. Par ailleurs, la déforestation de la forêt amazonienne, loin d'être liée à l'exploitation de la canne à sucre, renvoie à l'avancée d'un front pionner sous la pression des exploitants de bois et des éleveurs qui transforment progressivement la forêt en une savane packagée. Et c'est seulement ensuite que surviennent les cultures. Autrement dit, le sort de la forêt est avant tout un problème de volonté politique (ou de nonvolonté politique) du gouvernement brésilien sans liaison directe avec le développement de la canne à sucre.

Aux États-Unis la production d'éthanol à partir de maïs a prélevé environs 50 Mt en 2006 - une quantité du même ordre de grandeur que les exportations de maïs des USA qui $n^{\prime}$ ont pas baissé. En fait les USA ont augmenté leur 


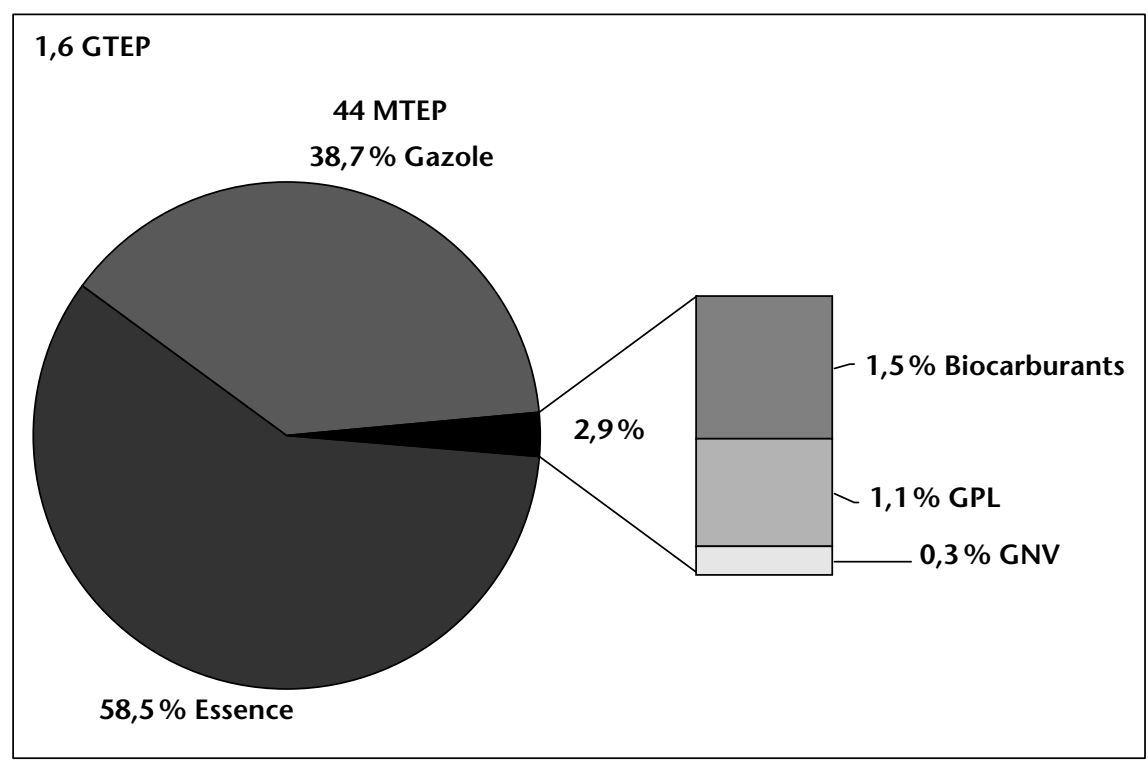

Figure 1. Carburants et consommation mondiale d'énergie dans le secteur des transports en 2005.

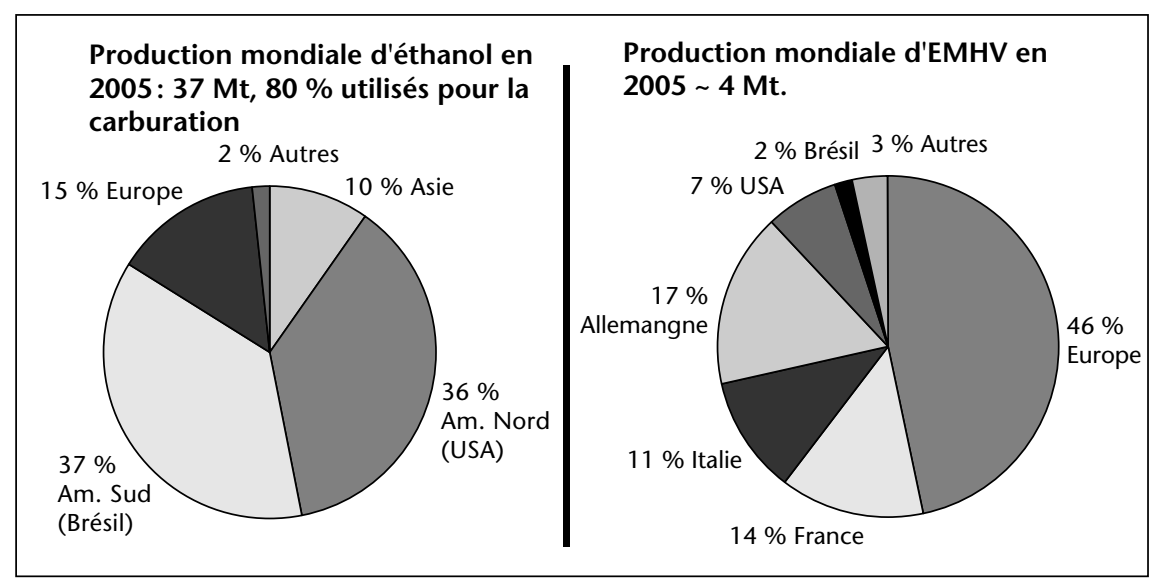

Figure 2. La production mondiale de biocarburants.

production sans se retirer du marché mondial. Et si le maïs est cher en Europe, on le doit aux OGM dans la mesure où les Européens ne veulent pas acheter du maïs OGM pour nourrir leurs animaux ${ }^{2}$. Ce segment du marché mondial du maïs connaît des prix évoluant de manière tout à fait différente que ceux du maïs et de l'éthanol dans l'Union européenne. Une interrogation demeure pour l'avenir devant la volonté américaine d'intensifier l'éthanol de maïs sans considération du marché mondial de I'alimentation animale.

\footnotetext{
$\overline{2}$ II ne faut pas oublier que la moitié des volumes de maïs utilisés pour produire de l'éthanol sont transformés en corn gluten feed et en drèches qui retournent à l'alimentation animale.
}

aussi bien qu'en volume, est mobilisé pour la production de biocarburants. À titre comparatif, on était à $30 \%$ en 2003 (figure 3).

\section{Rappel sur les biocarburants de première génération}

En matière de biocarburants il est plus que jamais nécessaire de pratiquer une analyse systémique d'intérêt.

Le sucre extrait de la betterave à sucre, une fois fermenté, produit de l'éthanol et les pulpes restantes peuvent être utilisées en alimentation animale.

De même, l'éthanol produit à partir de l'amidon extrait du blé, de la pomme de terre ou du maïs, laisse des drêches riches en protéines et pouvant être destinées à l'alimentation animale.

L'extraction de I'huile de colza, base des EMVH, donne lieu également à des co-produits, des tourteaux de colza également bons pour l'alimentation animale.

Tout le monde butte sur les co-produits en essayant de leur trouver une valeur énergétique au regard de la contrainte énergétique alors qu'ils ont un intérêt certain pour I'alimentation animale.

En 1991, I'Onidol a démontré à partir d'un modèle d'équilibre, l'intérêt de transformer I'huile de colza en EMHV pour la balance commerciale française et que le bénéfice résultait autant des économies d'importation de tourteaux de soja obtenues en leur substituant du tourteau de colza, que celles obtenues par la substitution des esters d'huile de colza à du carburant diesel.

Il est dommage que l'analyse des carburants de première génération ne soit faite qu'au regard de la contrainte énergétique. II faut également prendre en compte la contrainte en alimentation animale, qui est loin d'être négligeable et inintéressante, notamment du fait de notre déséquilibre en matières riches en protéines.

\section{Des évolutions de prix parallèles} d'huile végétale (EMHV) est quant à elle de $4 \mathrm{Mt}$, un chiffre dérisoire au niveau mondial. Cette production est essentiellement d'origine européenne, où ce volume devient significatif rapporté à la production de colza. Si bien qu'ici on peut identifier une plus grande liaison entre l'augmentation du prix de l'huile de colza et le développement des biocarburants pour les moteurs diesel.

L'examen des surfaces associées à la production des biocarburants en Europe permet de constater que, mis à part le colza, la part des surfaces pour le blé, le tournesol et la betterave sont tout à fait minimes. Ces surfaces ne sont significatives que pour le colza puisqu'en 2005 $49 \%$ du colza produit, en termes de surface
Il y a deux ans j'estimais que les biocarburants n'étaient pas encore rentables et qu'ils le deviendraient lorsque le pétrole sera autour de 85-90 \$ le baril. En fait les deux marchés ont évolué de la même façon : une demande mondiale forte pour l'énergie a coïncidé avec une demande mondiale forte pour les produits agricoles et alimentaires, si bien que l'huile de colza n'est toujours pas rentable aujourd'hui pour faire des biocarburants alors que les cours du pétrole ont dépassé 100 \$ le baril.

Et on assiste au même phénomène en ce moment aux États-Unis où certains projets en faveur de l'éthanol sont arrêtés et gelés avant $d^{\prime} y$ voir plus clair. 


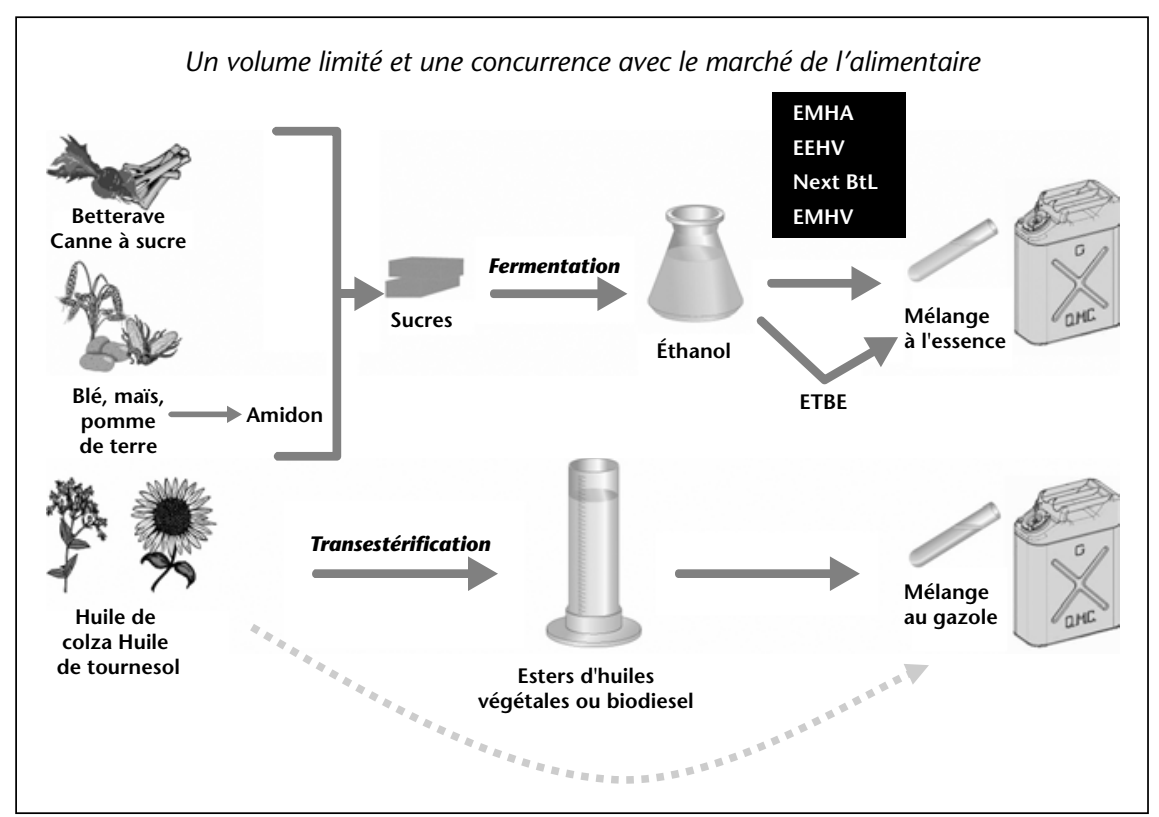

Figure 3. Les biocarburants de première génération.

Une partie du raisonnement sur le développement des biocarburants était bâtie sur l'idée que le prix de l'énergie devait monter et que le prix des produits agricoles devait baisser : ce qui devait rendre très intéressant cette solution. Il apparaît à la lumière des faits que le système ne se régule pas ainsi : on observe une interrelation entre les deux systèmes et pour l'instant les deux systèmes de prix évoluent en parallèle (figure 4). II n'y aura pas non plus une divergence constante entre l'évolution du prix de l'énergie et l'évolution du prix des produits agricoles servant de matières premières aux biocarburants.

En ce sens je n'ai pas une inquiétude particulière sur le fait d'une compétition durable entre les débouchés alimentaires et les usages non alimentaires de la production agricole, mis à part, pour l'instant, la détermination américaine à développer à tout prix la filière éthanol.

\section{Les carburants de deuxième génération}

Au lieu d'utiliser un sucre, un amidon ou une huile, les carburants de deuxième génération sont fondés sur l'utilisation de la plante entière, principalement constituée de lignine, d'hémicellulose et de cellulose.

L'intérêt est double: réclamant moins $d^{\prime}$ intrants agronomiques que les plantes

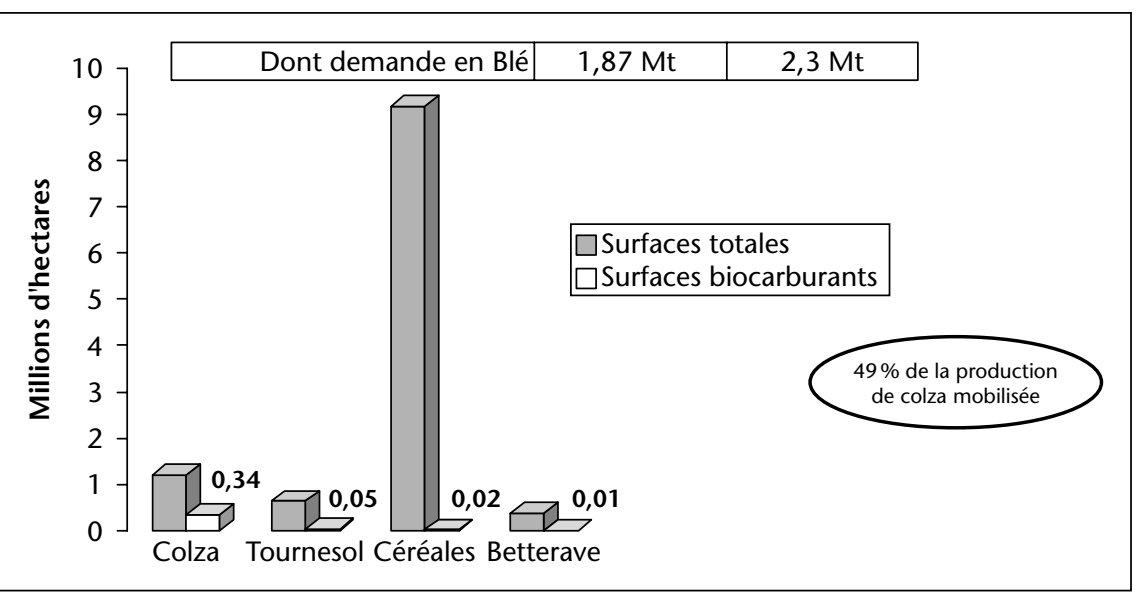

Figure 4. La biomasse « carburant » en Europe. Sources : FAO, FAPRI, USDA. annuelles ( $s^{\prime}$ accompagnant donc d'un moindre impact environnemental), les plantes en particulier ligno-cellulosiques permettent d'obtenir un rendement énergétique de 8 à 10 (contre 2 et 4 pour la première génération).

Tous les avantages donc semblent réunis.

Deux voies sont possibles:

- la voie thermochimique ou voie de la gazéification: en s'appuyant sur la réaction de Fischer-Tropsch et à partir des gaz produits on synthétise des carburants selon la longueur des chaînes soit pour l'essence, soit pour le gas-oil, soit pour les avions (figure 5);

- la voie enzymatique (la voie dont tout le monde rêve) fondée sur le " génie biochimique » et qui consiste à séparer la lignine de la cellulose et à opérer une hydrolyse enzymatique de la cellulose et des hemicelluloses permettant la production de sirops de sucres fermentescibles (sucres en $\mathrm{C} 6$ et en $\mathrm{C} 5$ ) $^{3}$.

Ces techniques sont très séduisantes: elles impliquent toutefois un saut technologique et biotechnologique important. En particulier, les enzymes cellulolytiques connues aujourd'hui sont relativement spécifiques, et l'on ne dispose pas encore de systèmes plus polyvalents, moins dépendants de tel ou tel type de substrat (figure 6).

Cette voie est une très belle perspective qui débloquerait la pression sur les productions alimentaires traditionnelles en recourant à la production d'énergie à partir d'une matière première lignocellulosique. Mais elle ne peut être considérée pour l'instant que comme une solution à moyen terme (de sept à quinze ans selon les auteurs).

Le passage de la première à la seconde génération en matière de biocarburants devrait se faire progressivement : on commencera en fait, comme le font actuellement les Brésiliens, par digérer la cellulose - le rendement énergétique de la canne à sucre est ainsi amélioré en extrayant la partie purement cellulosique de la bagasse, afin de produire par voie enzymatique des sucres fermentescibles pour faire de l'éthanol. La voie vers la deuxième génération devrait se faire à mon avis par l'intermédiaire du traitement enzymatique de co-produits de la première génération. Par la suite, il faudra déterminer les cultures et les productions ligneuses les mieux adaptées, en n'oubliant pas I'optimisation des questions de logistique qui supposent une certaine concentration de la production autour des usines de transformation.

$\overline{3}$ La fermentation des sucres en C5 suppose également le recours à des travaux de génie moléculaire. 


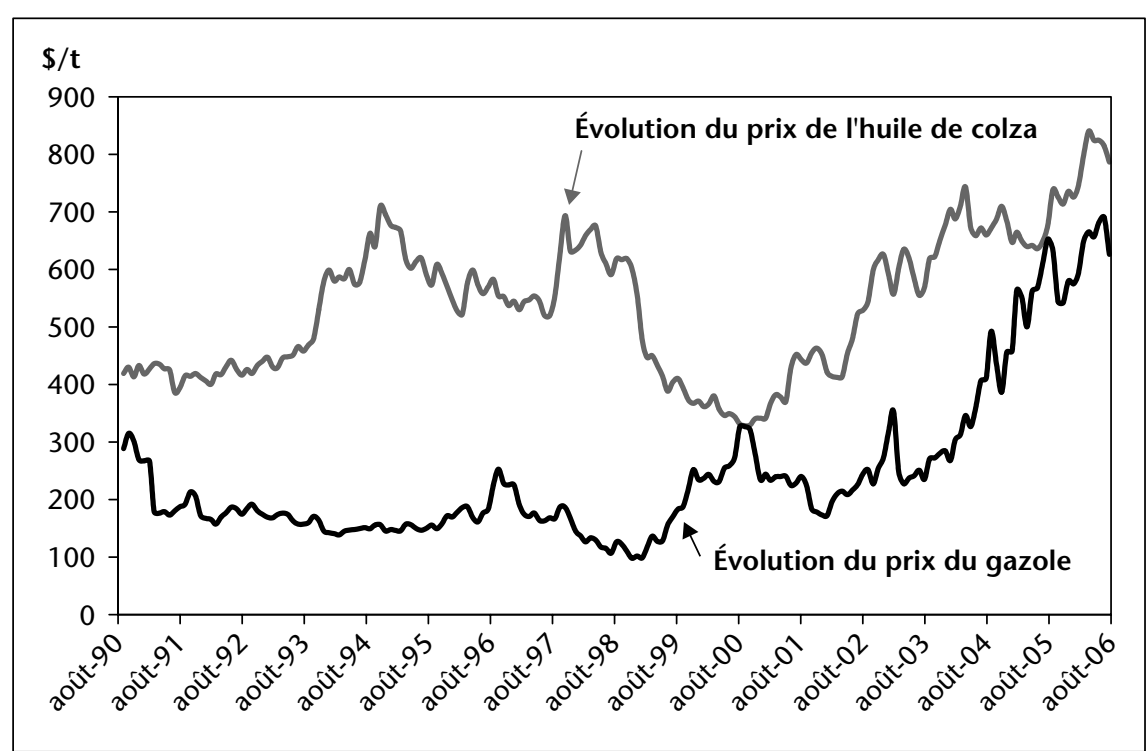

Figure 5. Évolution comparée du prix d'huile de colza et du gazole en Europe.

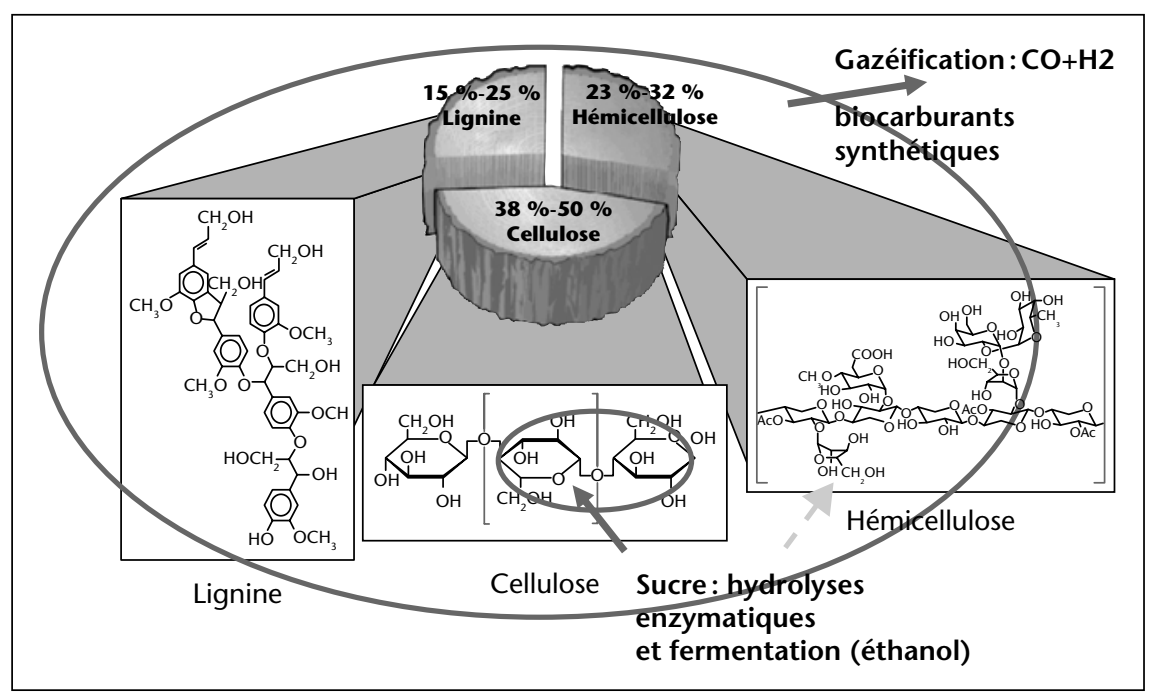

Figure 6. Les nouvelles filières de biocarburant.

\section{Conclusion}

Je rappellerai pour conclure qu'il convient de ne pas confondre volatilité des marchés et désajustement structurel. Les problèmes actuels de volatilité ne marquent pas à mon avis le début de la crise de l'énergie et de l'agriculture. Les surfaces agricoles consacrées aux carburants de première génération s'élèvent à quelques pourcents de la surface totale pour l'agriculture. Elles ont quand même un effet tant sur l'agriculture que sur le marché énergétique, dans la mesure où elles contribuent à limiter la volatilité des marchés pétroliers.

L'autre point intéressant de la politique en en faveur des biocarburants, indépendamment de leur impact sur l'effet de serre, c'est que dans un contexte d'abandon des politiques de soutien des marchés agricoles, un certain nombre de pays se résolvent à maintenir des politiques publiques en faveur de l'agriculture au travers de politiques volontaristes en matière de biocarburants.

Cette politique de valorisation de la biomasse est un moyen indirect de régulation des marchés agricoles, ce qui peut être intéressant pour la majorité des agriculteurs pauvres. Un soutien des cours des produits agricoles me paraît plus bénéfique pour l'avenir de la planète que leur effondrement, pour autant que le développement des cultures destinées à la production d'énergie ne perturbe pas la reconstruction de stocks de régulation des marchés agricoles qui s'impose aujourd'hui comme une nécessité impérative.

Et dernier point de ma conclusion, il y a nécessité dans ce domaine (comme dans bien d'autres) de travaux de recherche et de développement importants car le passage de la première à la deuxième génération ne pourra être que progressif et exigera encore de nombreuses expérimentations en vraie grandeur. 